\title{
A new Approach in Designing Low Vision Aids (LVA)
}

\author{
Berry P.L.M. den Brinker \\ Department of Psychology \\ Faculty of Human Movement Sciences \\ Vrije Universiteit van Amsterdam \\ van de Boechorstraat 9 \\ 1081 BT Amsterdam, The Netherlands
}

\begin{abstract}
In reading text, two processes can be discriminated: 1) the search for desired information on the page, and 2) the process of processing information once the desired spot on the page has been found. A global overview is given about the research on the structure of the visual system and how this system is used in normal reading and reading by people with low vision (LVP). It is concluded that research has been concentrated at the second process of the reading process and that no knowledge is available on how LVP can use their residual visual function to enable search. It is argued that visual search is of increasing importance in our modern society and that low vision aids (LVA) should support visual search. It is assumed that betters LVA can be developed on the basis of an 'Ecological approach to visual perception' (Gibson (1979).
\end{abstract}

\section{Introduction}

Today, our modern society requires more processing of visual information than ever before. Visual information is presented in various ways via books, papers, magazines, television, high definition computer monitors, LCD control panels, and so on. The expanding flow of visual information places an increasing demand on the visual system in occupational as well as daily life situations. As a consequence, people with reduced vision will be confronted more quickly with their disabilities, which can result in a handicap. More than in the past, rehabilitation is aimed at using the potentials of the visually impaired person (Welling, 1994).

In training low vision people (LVP) a shift has taken place from substitutional/compensatory training to utilisation of the residual vision (Backman, 1994). From the 1970es onward LVP are trained to use magnifiers and other low vision aids (LVA) to enable or support reading. It no longer necessary to blindfold LVP during Braille training. New electronic devices are developed, the most prominent being the closed circuit television (CCTV) magnifier and programs to enlarge the characters on the computer screen (LEP). Nowadays the use of these LVA is wide spread in occupational situations and they are also becoming more and more popular in daily life situations. Moreover, the need for LVA will grow because the number of age related cases of low vision will increase in the near future. 
However, there are several reasons to reconsider the design of reading aids. First of all reading with a CCTV magnifier is slow, and even more important, tiring. As a consequence, LVP tend to avoid its use (den Brinker e.a., 1994) or stop using it at all (Buijk, 19986). Secondly, the present generation of letter enlarge programs (LEP) is becoming out-of-date since the introduction of graphical oriented window systems (Vanderheijden, 1989).

Problems connected with the LVA can be solved by determining in practice what the complaints are and engage in a trial-and-error procedure for minimizing inconveniences for the actual users. This approach is at present indispensable. However, good engineering should rest on solid insight into the functions that the to be developed devices would have to fulfil to avoid unacceptable situations (Bouma, 1980). Therefore insight in the reading process and visual search is necessary for designing LVA.

In this presentation an overview is given of the reading process as it occurs in normal-sighted and low vision people. It is followed by a critical look at the design of the presently available electronic devices for reading and computer use. Finally, suggestions are given about tests to be developed for evaluating reading performances in both normal and low vision people.

\section{The Visual System}

From a biological perspective, the visual system has to support the organism in the basic functions of navigation and object-recognition under varying conditions of illumination. These tasks led to conflicting demands for the 'biological engineer'. For example, the requirements of high acuity and high sensitivity cannot be met simultaneously. The present situation, with a small retinal region of high acuity and a decreasing acuity with eccentricity is a good compromise between acuity and sensitivity: an eye with full retinal acuity would require 150 times more fibres and thus lead to an unacceptable large blind spot (Van der Grind, 1994). The lack of full retinal acuity is compensated for by an ingenuous control system by which all parts of the surrounding world can be focused into the central region (fovea and parafovea). In other words, it is the combination of a small retinal region of high acuity and a eye movement control system that enables us to get sharp images of every detail in our visual environment.

\section{Normal Reading}

In reading texts two processes can be distinguished (Bouma, 1980; Van Nes, 1986). First the search for desired information on the page, and second, the processing of that information. To optimize legibility (the extend to which visual information can be processed during reading) the structure of the page should enable rapid search and subsequent reading of the interesting passages. While searching, the eyes scan the page, guided by text attributes such as words that have specific first letters, length or printing style (bold face), etc or whole areas that are conspicuous by there layout.

During reading of connected text the eyes make rapid jumps, saccades. Between saccades, the fixation pauses, information is extracted from the visual reading field 
(about 10 to 20 characters). During this process both letter recognition and word contour recognition play a role. Words in lower case have a high legibility because of characteristic contours from ascenders $(b, \mathrm{~d}, \mathrm{f}, \mathrm{h}, \mathrm{k}, \mathrm{l}, \mathrm{t})$ and descenders $(\mathrm{g}, \mathrm{j}, \mathrm{q}, \mathrm{y})$. While reading the eyes proceed along the line with rightward saccades of 4 to 12 characters, the length being determined by both display and content variables. This leads to the typical 'staircase' pattern of eye movements (see Figure 1). Small backward or correction saccades are made when comprehension of the text lags behind the recognition process during the fixation pauses. Line saccades, long leftward eye jumps, are made when the end of a line is reached. During a line saccade, the beginning of the next line has a peripheral retinal projection and therefore cannot be seen clearly. Hence, line saccades are often misdirected. Therefore the left margin should be a straight line and sufficiently wide to prevent under- and overshoots in horizontal direction. Errors in vertical direction occur when the angle between the line saccade and the line is to small (less than 2 degrees), which happens when lines are to long or to close.

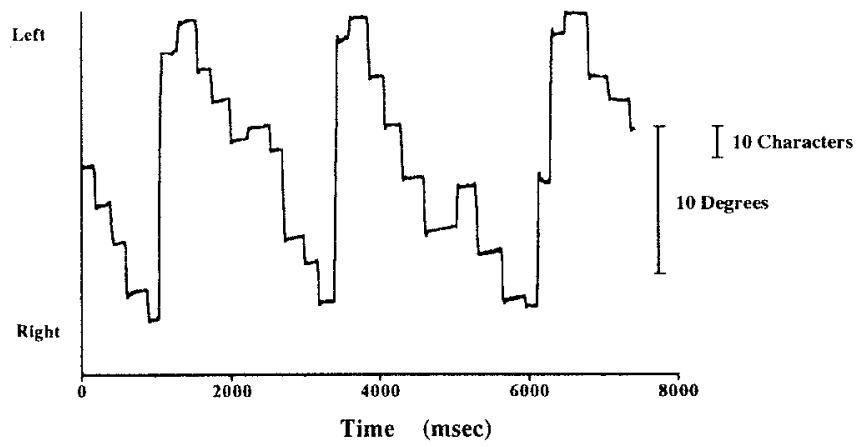

Fig. 1. A typical 'staircase' pattern of eye movements in normal reading. (Adopted with permission from Bowers \& Ackerley, 1994)

\section{Reading with Magnifiers}

Perception can not be studied without reference to movements. As shown above, this is also the case for 'pure' perceptual tasks such as reading. For people with reduced vision movement control is even more important. While reading, they have to hold the magnifier and synchronize the eye movements with those of the magnifier. Little is known about the particular difficulties of reading with a loupe compared to normal reading. Investigations should be directed at the effect of magnification, reading-field width, lens aberration, reflection of lens-surface, and how these factors relate to the control of the movements of the eyes and loupe. The study of Blomnaert \& Neve (1987) shows the complexity of these problems. They calculated the theoretical maximum reading-field width of three kinds of looking through loupes (monocular, binocular and 
a composite method). Experimentally they tried to identify these styles and effective field widths in five normal subjects and in one subject with moderate low vision. All three styles were identified and the effects were discussed in relation to other parameters, such as spherical and chromatic aberrations and reflection. It was assumed that loupes should not be used as eye glasses and that for severely impaired readers the absence of aberration is more important than field of view or magnification. These ideas, described in an information brochure (Korpel, Tebak \& de Vette, 1989), are contrary to what is sometimes found in practice. People with poor visual acuity often prefer to use loupes as eye glasses to compensate for the severe loss of the reading-field width caused by the small diameter of the strong loupe. Furthermore, it can be shown (see below) that aberrating lenses, when used as eye glasses, need not disturb the perception process.

An important other class of magnifiers is that of the closed-circuit television magnifier, CCTV magnifier or video-loupe. Studies about the field of view, magnification, contrast, polarity of the magnified image, etc are easier to be carried out using these electronic devices. However, the quality of the electronic parts may have an aversive effect on the observed behaviour and makes it difficult to draw general conclusions. This is exemplified by the research of Lowe \& Dresdo (1990). While discussing the optimal strategy for moving the text (on a X-Y platform) along the camera, they speculated that it is better not to move the X-Y platform during the recognition phase to prevent blurring of the image (p.232). This would not be a good advise using modern CCTV magnifiers. Their blur was caused by the inferior quality of camera tubes that were used at the time of their experiments (before 1980). Modern CCTV magnifiers, equipped with a CCD camera, don't have this problem and enable the same movement patterns (eye and text) as those found with loupes.

From the literature it is clear that LVP don't have the typical 'staircase' pattern of eye movements. While moving the magnifier smoothly along the line, they fixate on the same location in the image. As soon as the recognition process is completed, the eye jumps to the next fixation point in the moving image (opto-kinetic nystagmus: OKN) (see Figure 2). The resultant 'saw-tooth' pattern has been found in LVP (Legge e.a., 1985; Legge, 1991) and in normal-sighted subjects using magnifiers (Fotinakis \& Dickinson, 1994).

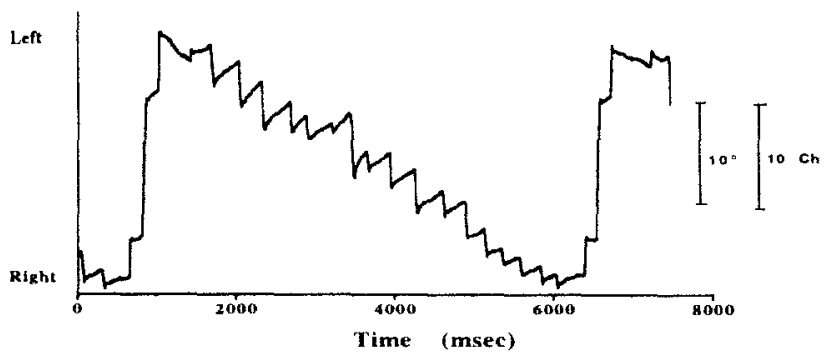

Fig. 2. A typical 'saw-tooth' pattern of eye movements while reading with a hand hold magnifier. (Adopted with permission from Bowers \& Ackerley, 1994) 
Efforts have been made to find parameters that determine reading performance using LVA (hand-hold magnifiers, spectacle-mounted magnifiers and CCTV magnifiers). In general, character size (or magnification) was found to be negatively correlated with reading rate (Fotinakis \& Dickinson, 1994; Lowe \& Drasdo, 1990; Legge, 1985). However there is no agreement on the minimal number of characters in the viewing field. Legge e.a. (1985) found optimal reading with a viewing window of only 4 characters, while Lowe (\& Drasdo, 1990) claimed 20 to 30 were necessary.

In any case, the two parameters are representative for the research into the use of LVA; all research is directed at parameters that are directly or indirectly responsible for the quality of the display. The relevance of these parameters is determined on the basis of the reading rate of a continuous text of 100 to 200 words. By doing so, no information is collected about the way LVA are used for the search process, which was distinguished by Bouma (1980) as one of the two basic ingredient of the reading process.

\section{The Ecological Validity of Reading rate Tests}

In a recent review on the comparison of reading from paper and screens, Dillon (1992) criticized the use of reading rate as a test for reading performance. Such tests are based on the assumption that textual information consists of a sequence of words that has to be recognized and comprehended in a fixed order. In real life, that is only the case with prose. Papers, magazines, telephone book, manuals, flyers, study books, etc, are not meant to be read from the beginning to the end. They are all designed to help the reader to find the desired information. Books have contents, index registers, glossaries, etc to help the reader to find his way through the pages. As soon as the reader has reached the page on which he expects to find information, the layout may help him find it.

As mentioned in the introduction, our modern society requires its participants to digest loads of visual information. The search for desired information is at least as important as the processing of that information. So, if we want to support people with reduced vision, we have to help them find that information. The equipment that has been developed so far was not designed for that purpose. It's suitable for processing the information that was found otherwise. In a recent study (den Brinker e.a., 1994) 22 LVP were interviewed about activities of daily live. None of the interviewed CCTV magnifier owners used the apparatus for tasks that require search (numbers in a telephone book). It is almost a tragedy that the present CCTV magnifiers only support the part of text processing that probably will be replaced in the future by speech-generation (on the basis of opto-electronic character recognition).

\section{Future Developments in Research and Design}

In the last fifteen years a correspondence is found between research in (the improvement of) the quality of video display units (VDU) and research in the use of low vision aids. In both research lines display characteristics are of major concern and were tested with the same kind of reading performance tasks.

Dillon (1992) was one of the first authors who questioned the importance of 
manipulation of the text in determining the usability of computerized information systems. Most people still prefer books over computerized information systems in which people easily get lost. In discussing the usability the concept of the 'navigation problem' is often mentioned as the major factor determining the unpopularity of hyper text based information systems.

LVP using LVA have similar problems. While reading with a magnifier, the biggest part of their visual field is filled with only a few words. During the search for desired information they have to switch back and forth between an overview and an enlarged image. This process is made even more complex by the need to refocus the magnifier when the search is shifted to another part of the page or the book.

It is clear that something has to be done to attack the problem of manipulation and navigation. It is wise not to start with a trial and error search for parameters that may influence the usability of modern information systems. I have the idea that there is a correspondence between navigation in 'the real world' and navigating in information systems, virtual reality, and the use of CCTV magnifiers. Therefore the work on locomotion and manipulation by Gibson (1979) is very relevant for research in ergonomics.

His 'Ecological approach to visual perception' makes clear that perception and action have to be considered complementary, tightly coupled subsystems of the perceptual system. Gibson rejects the idea that it is possible to understand how we move around in an environment, by considering the eyes as cameras, connected by nerves to transport images to the brain. Instead, he suggests that natural vision depends on the eyes in the head on a body supported by the ground with the brain as the centre of the visual system. He describes how such a system is able to detect the invariant aspects in the ambient optic array necessary to distinguish changes in the environment from changes caused by eye, head and body movements. The sequence of stimulation is not a sequence of discrete snapshots, but a sequence that is converted into a (stable) scene. The optical flow is continuously sampled by the visual system and the invariants contribute to a persistent environmental scene in which co-existing parts (objects) and events are all perceived together (p. 222). With such a system it can be understood that our visual system interprets straight lines as such, although the retinal projection of that line isn't linear at all.

With this theory in mind an earlier presented problem in the use of lenses can be understood. When handling a loupe with spherical aberrations on some distance of the eye, conflicting images will emerge: the lines seen through the loupe are more or less curved while the same lines outside the border of the loupe remain straight. This situation will disturb the reading process as was stated by Blomnaert \& Neve (1987). When using the same loupe as an eye glass these image distortions will, after a while, not be noticed. While moving the head along the lines of the text, the eye glass will consequently represent the lines as curves and still be interpreted as straight lines because of the absence of conflicting visual images.

This is only a minor example of how Gibson's theory may contribute to the understanding of the problems that LVP face when handling LVA. If, as I have said before, a major problem for LVP in our modern society is to find the desired information, we have to know more about visual search and navigation by those people. 
If navigating in an environment, according to Gibsons view, can be described as a continuous extraction of invariant features from the ambient optical flow to contribute to the building of a permanent scene, one might imagine that the use of some LVA may disturb this process of the visual system. Since we know from the literature (Kooijman e.a. 1994) that LVP are able to navigate in their living environment in spite of major losses of visual acuity, there are reasons to believe that tools can be developed to profit from this capability while searching for desired information to read.

When designing new aids for LVP, the engineer has to know which cues, to state it in more traditional terms, are available for people with reduced vision to support him in the search for desired information. Finally, more relevant, ecological valid assessments of the usability have to be developed as well.

\section{References}

1. O. Backman: Reading skills, reading training and technology for the visually handicapped, Prospects for the 1990 es. In: A.C. Kooijman, P.L. Looijstijn, J.A. Welling \& G.J. van der Wildt (eds): Low Vision, Research and new developments in rehabilitation. Amsterdam: IOS Press 1994, 251-254

2. J.J. Blomnaert, J.J, Neve, J.J.: Reading fields of magnifying loupes. Journal of the Optical Society of America 4, 1820-1830 (1987)

3. H. Bouma: Visual reading processes and the quality of text displays. In: E. Grandjean, E. Vigliani: Ergonomic aspects of Visual Display Terminals. London: Taylor \& Francis Ltd 1980, 101-114

4. A. Bowers, R. Ackerly: Reading characteristics of normal observers using Low Vision Aids. In: A.C. Kooijman, P.L. Looijstijn, J.A. Welling, G.J. van der Wildt (eds.): Low Vision, Research and new developments in rehabilitation. Amsterdam: IOS Press 1994, 235-238

5. B.P.L.M. den Brinker, K. Bolder, K. Mollevanger, P. Tummers: The use of Low Vision Aids (LVA) in daily life activities. In preparation (1994)

6. C.A. Buijk: Slechtziendheid en hulpmiddelen. Amsterdam: LTP 1986

7. A. Dillon: Reading from paper versus screens: a critical review of the empirical literature. Ergonomics 35, 1297-1326 (1992)

8. V.P. Fotinakis, C.M. Dickinson: Reading with Hand Magnifiers. In: A.C. Kooijman e.a. (eds.): Low Vision, Research and new developments in rehabilitation. Amsterdam: IOS Press 1994, 259-268

9. J.J. Gibson: The ecological approach to visual perception. Boston: Houghton Mifflin Company 1979

10. W.A. van de Grind: Limits and mechanisms of vision. In: A.C. Kooijman e.a. (eds): Low Vision, Research and new developments in rehabilitation. Amsterdam: IOS Press 1994, 28-37

11. A.C. Kooijman, G.I.J.M. Kempen, F.W. Cornelissen, M.J.G. Heuvelen, A. van de Wege, P. Fritsche, W.A. Houtman: Screening of visual function compared with self-report visual disability. In: A.C. Kooijman, P.L. Looijstijn, J.A. Welling, G.J. van der Wildt (eds.): Low Vision, Research and new 
developments in rehabilitation. Amsterdam: IOS Press 1994, 11-14

12. L.M.H. Korpel, N.E. Tebak, M.E. de Vette: Met een loep pakt u de draad weer op! Eindhoven: IPO 1989

13. G.E. Legge, C.S. Rubin, D.G. Pelli, M.M. Schleske: Psychophysics of ReadingII, Low Vision. Vision Research 25, 253-266 (1985)

14. G.E. Legge: Glenn A. Fry Award Lecture 1990: Three Perspectives on Low Vision Reading. Optometry and Vision Science. American Society of Optometry, 763-769 (1991)

15. J.B. Lowe, N. Dresdo: Efficiency in reading with closed-circuit television for low vision. Ophthalmology Physiological Optics 10, 225-233 (1990)

16. F.L. van Nes: Space, Color and typography on visual display terminals. Behaviour and Information technology 5, 99-118 (1986)

17. C.C. Vanderheijden: Nonvisual alternative display techniques for Output of Graphics-Based-Computers. Journal Of Visual Impairment and Blindness 83, 383-390 (1989)

18. J.A. Welling: Foreword. In: A.C. Kooijman, P.L. Looijstijn, J.A. Welling, G.J. van der Wildt (eds.): Low Vision, Research and new developments in rehabilitation. Amsterdam: IOS Press 1994, XI-XII 